\title{
Tethered Spinal Cord Syndrome
}

National Institute of Neurological Disorders and Stroke (NINDS)

\section{Source}

National Institute of Neurological Disorders and Stroke (NINDS). Tethered Spinal Cord

Syndrome Information Page.

Tethered spinal cord syndrome is a neurological disorder caused by tissue attachments that limit the movement of the spinal cord within the spinal column. Attachments may occur cong enitally at the base of the spinal cord (conus medullaris) or they may develop near the site of an injury to the spinal cord. These attachments cause an abnormal stretching of the spinal cord. The course of the disorder is progressive. In children, symptoms may include lesions, hairy patches, dimples, or fatty tumors on the lower back; foot and spinal deformities; weakness in the legs; low back pain; scoliosis; and incontinence. This type of tethered spinal cord syndrome appears to be the result of improper growth of the neural tube during fetal development, and is closely linked to spina bifida. Tethered spinal cord syndrome may go undiagnosed until adulthood, when pain, sensory and motor problems, and loss of bowel and bladder control emerge. This delayed presentation of symptoms is related to the degree of strain placed on the spinal cord over time and may be exacerbated during sports or pregnancy, or may be due to narrowing of the spinal column (stenosis) with age. Tethering may also develop after spinal cord injury and scar tissue can block the flow of fluids around the spinal cord. Fluid pressure may cause cysts to form in the spinal cord, a condition called syringomyelia. This can lead to additional loss of movement, feeling or the onset of pain or autonomic symptoms. 